\title{
Real-time reverse transcription loop-mediated isothermal amplification for rapid detection of SARS-CoV2
}

\author{
Yee Ling Lau ${ }^{\text {Corresp., }}{ }^{1}$, Ilyiana binti Ismail ${ }^{2}$, Nur Izati binti Mustapa ${ }^{2}$, Meng Yee Lai ${ }^{1}$, Tuan Suhaila binti Tuan Soh ${ }^{2}$, \\ Afifah Haji Hassan ${ }^{2}$, Kalaiarasu M Peariasamy ${ }^{3}$, Yee Leng Lee ${ }^{3}$, Yoong Min Chong ${ }^{4}$, I-Ching Sam ${ }^{4}$, Pik Pin Goh $^{5}$ \\ ${ }^{1}$ Department of Parasitology, Faculty of Medicine, University of Malaya, Kuala Lumpur, Malaysia \\ Department of Pathology, Hospital Sungai Buloh, Sungai Buloh, Selangor, Malaysia \\ 3 Clinical Research Centre, Hospital Sungai Buloh, Selangor, Sungai Buloh, Malaysia \\ 4 Department of Medical Microbiology, Faculty of Medicine, University of Malaya, Kuala Lumpur, Malaysia \\ 5 Institute of Clinical Research (ICR), National Institutes of Health, Ministry of Health Malaysia, Putrajaya, Malaysia \\ Corresponding Author: Yee Ling Lau \\ Email address: lauyeeling@um.edu.my
}

Background. Highly sensitive real-time reverse transcription polymerase chain reaction (qRT-PCR) methods have been developed for the detection of SARS-CoV2. However, they are costly. Loop-mediated isothermal amplification (LAMP) assay has emerged as a novel alternative isothermal amplification method for the detection of nucleic acid.

Methods. A rapid, sensitive, and specific real-time reverse transcription LAMP (RT-LAMP) assay was developed for SARS-CoV2 detection.

Results. This assay detected 1 copy of SARS-CoV2 RNA in 30 mins. Both the clinical sensitivity and specificity of this assay were $100 \%$. The RT-LAMP showed comparable performance with real-time qRTPCR. Combining simplicity and cost-effectiveness, this assay is therefore recommended for use in resource limiting settings. 


\section{Real-Time Reverse Transcription Loop-Mediated}

\section{Isothermal Amplification for Rapid Detection of SARS-}

\section{CoV2}

4

5 Yee Ling Lau ${ }^{1 *}$, Ilyiana binti Ismail ${ }^{2}$, Nur Izati binti Mustapa ${ }^{2}$, Meng Yee Lai ${ }^{1}$, Tuan Suhaila

6 binti Tuan Soh², Afifah Haji Hassan², Kalaiarasu M Peariasamy ${ }^{3}$, Yee Leng Lee ${ }^{3}$, Yoong Min

7 Chong ${ }^{4}$, I-Ching Sam ${ }^{4}$, Pik Pin Goh ${ }^{5}$

$8{ }^{1}$ Department of Parasitology, Faculty of Medicine, University of Malaya, Kuala Lumpur,

9 Malaysia

$10 \quad{ }^{2}$ Department of Pathology, Hospital Sungai Buloh, Selangor, Malaysia

$11{ }^{3}$ Clinical Research Centre, Hospital Sungai Buloh, Selangor, Malaysia

$12{ }^{4}$ Department of Medical Microbiology, Faculty of Medicine, University of Malaya, Kuala

13 Lumpur, Malaysia

$14{ }^{5}$ Institute of Clinical Research (ICR), National Institutes of Health, Ministry of Health Malaysia, 15 Putrajaya, Malaysia

17 Corresponding Author:

18 Yee Ling Lau

19 Department of Parasitology, Faculty of Medicine, University of Malaya, 50603 Kuala Lumpur, 20 Malaysia.

21 E-mail: lauyeeling@um.edu.my 


\section{Abstract}

24 Background. Highly sensitive real-time reverse transcription polymerase chain reaction (qRT25 PCR) methods have been developed for the detection of SARS-CoV2. However, they are costly. 26 Loop-mediated isothermal amplification (LAMP) assay has emerged as a novel alternative 27 isothermal amplification method for the detection of nucleic acid.

Methods. A rapid, sensitive, and specific real-time reverse transcription LAMP (RT-LAMP) assay was developed for SARS-CoV2 detection. Forty-seven qRT-PCR positive and 42 qRT-PCR negative nasopharyngeal swabs samples were tested.

Results. This assay detected one copy of SARS-CoV2 RNA in 30 mins. Both the clinical sensitivity and specificity of this assay were 100\%. The RT-LAMP showed comparable performance with real-time qRT-PCR. Combining simplicity and cost-effectiveness, this assay is therefore recommended for use in resource limiting settings.

\section{Introduction}

A novel coronavirus, SARS-CoV2, was recently identified causing pneumonia in humans, termed coronavirus disease 2019 (COVID-19). Cases of this new infection were first reported in Wuhan, China in December 2019, and the outbreak spread to more than 155 other countries in a short time [1]. The World Health Organization (WHO) declared the COVID-19 outbreak as a pandemic on

11 March 2020. Several real-time reverse transcription polymerase chain reaction (qRT-PCR) methods have been developed and recommended by Centers for Disease Control (CDC) of the United States [2] and WHO [3,4] for detection of SARS-like coronaviruses and specific detection of SARS-CoV2. These methods are highly sensitive and specific but are too expensive to be widely used in many developing countries. Real-time RT-PCR also requires experienced personnel, 
46 maintenance of reagents in cold storage facility, and use of a high-precision thermal cycler. Loop-

47 mediated isothermal amplification (LAMP) has emerged as a novel alternative isothermal

48 amplification method for the detection of nucleic acid [5]. The LAMP assay was reported to take

49 less than one hour to perform at a constant temperature. Since LAMP does not require any major

50 equipment and is simple to perform, it represents an ideal diagnostic tool for use in areas with

51 limited resources. LAMP assays have been described for the detection of various other infectious

52 agents including dengue viruses [6,7] and malaria parasites [8]. In order to further reduce costs

53 and enable detection by the naked eye, we used hydroxynaphthol blue (HNB) dye for the

54 colorimetric detection of the amplification reaction. To our knowledge, this is the first report of

55 the detection of SARS-CoV2 by real-time reverse transcription LAMP (RT-LAMP) assay with

56 HNB.

57

\section{Materials \& Methods}

\section{RT-LAMP assay}

Primers were designed using the Primer-Explorer V4 software (Eiken Chemical Co., Ltd., Tokyo, Japan) based on SARS-CoV2 nucleoprotein (GenBank accession no MN988713.1, LC528233.1 and MT123293.1). The sequences of the selected primers were conserved among the SARS-CoV2 sequences (Supplementary Figure 1) that do not share homology with other SARS-like coronavirus and Middle East respiratory syndrome coronavirus (MERS) sequences (Supplementary Figure 2). Reverse transcription LAMP (RT-LAMP) was carried out using Loopamp RNA amplification kit (Eiken Chemical Co., Ltd., Japan). The amplification was carried out in a Loopamp real-time turbidimeter (LA-320; Teramecs, Co., Ltd., Tochigi, Japan) at $65^{\circ} \mathrm{C}$ for 30 min with $2 \mathrm{X}$ reaction mixture, $5 \mu \mathrm{L}$ RNA template, and species-specific primers. The primer sequences are listed in 
69 Supplementary Table 1. Endpoint assessment was done by visual inspection following the addition

70 of $1 \mu \mathrm{HNB}$ (Sigma, USA) in the master mix; a positive amplification was indicated by a colour

71 change from violet to sky blue (Figure 1).

\section{Analytical sensitivity and specificity}

74 To determine the analytical sensitivity of the SARS-CoV2 RT-LAMP assay, in vitro transcript

75 RNAs were prepared using a previously published method [9]. Briefly, target gene fragments were

76 cloned to a pGEM-T vector as described in the manufacturer's protocol (Promega, USA).

77 Following digestion with SalI restriction endonuclease (NEB, USA), the plasmids were purified.

78 In vitro transcribed RNAs were prepared with RiboMAX ${ }^{\mathrm{TM}}$ System (Promega, USA) and digested

79 by deoxyribonuclease (DNase) I as described in the manufacturer's protocol. The product was then

80 purified by phenol-chloroform extraction method. Finally, in vitro transcribed RNAs were

81 quantified by UV spectrophotometry. The limit of detection (LOD) was determined using 10-fold

82 serially diluted in vitro transcript RNA with known numbers of nucleic acid copies $(10 \mathrm{cp} / \mu \mathrm{L}, 5$

$83 \mathrm{cp} / \mu \mathrm{L}, 2 \mathrm{cp} / \mu \mathrm{L}, 1 \mathrm{cp} / \mu \mathrm{L}$ and $0.1 \mathrm{cp} / \mu \mathrm{L})$ and comparing the assay with qRT-PCR. The reactions

84 were carried out in duplicates. One $\mu \mathrm{L}$ of each diluted in vitro transcript RNA was used for qRT-

85 PCR and RT-LAMP assay.

86 The specificity of the RT-LAMP assay was determined by using genomic RNA of coronaviruses

87 (HCoV-OC43 and SARS-CoV), adenovirus, human metapneumovirus, influenza A

88 (A/H1pdm2009 and A/H3) viruses, influenza B virus, parainfluenza virus 3, rhinovirus A, 89 respiratory syncytial virus B and enterovirus D68.

90

\section{Clinical sensitivity and specificity}


92 Forty-seven qRT-PCR positive and 42 qRT-PCR negative nasopharyngeal swabs samples were 93 randomly chosen, regardless of qRT-PCR threshold cycle value (Ct-value) from a recent COVID-

9419 outbreak in Malaysia (2020) were collected by Hospital Sungai Buloh, Malaysia.

95 Nasopharyngeal swabs were collected using Dacron swabs made of polyester fiber. The swab was

96 inserted into the nostril and back to the nasopharynx and left in place for a few seconds then slowly

97 withdrawn with a rotating motion. The swab was immediately placed into a sterile vial containing

$982 \mathrm{ml}$ of viral transport media. Total RNA was extracted using QIAamp viral RNA Mini kit (Qiagen,

99 Germany) according to the manufacturer's instructions. In brief, $140 \mu \mathrm{L}$ of cell lysates were 100 transferred into $1.5 \mathrm{~mL}$ tubes containing $560 \mu \mathrm{L}$ of Buffer AVL followed by vortex for $15 \mathrm{~s}$. After 101 standing at room temperature for 10 minutes, the collection tube was briefly centrifugated. Then $102560 \mu \mathrm{L}$ of ethanol (96-100\%) was added to the sample, and mixed by vortex for $15 \mathrm{~s}$. The mixture 103 was then transferred to QIAamp Mini column and washed with Buffer AW1 and AW2. A $50 \mu \mathrm{L}-$ 104 elution was obtained for each sample. The RNA samples were analysed by qRT-PCR (Figure 2), 105 as previously described [3,4]. Briefly, $5 \mu \mathrm{L}$ of extracted RNA were added as template into $20 \mu \mathrm{L}$ 106 of the reaction mixture containing $12.5 \mu \mathrm{L}$ of $2 \times$ reaction buffer provided with the Superscript III 107 one step RT-PCR system with Platinum Taq Polymerase (Invitrogen, USA), $1 \mu \mathrm{L}$ of reverse 108 transcriptase/Taq mixture from the kit, $0.4 \mu \mathrm{L}$ of a $50 \mathrm{mM}$ magnesium sulphate solution and $1 \mu \mathrm{g}$ 109 of nonacetylated bovine serum albumin. Primer and probe sequences targeted at RdRP and E genes 110 were used. Thermal cycling was performed at $55^{\circ} \mathrm{C}$ for $10 \mathrm{~min}$ for reverse transcription, followed 111 by $95^{\circ} \mathrm{C}$ for 3 min and then 45 cycles of $95^{\circ} \mathrm{C}$ for $15 \mathrm{~s}, 58^{\circ} \mathrm{C}$ for $30 \mathrm{~s}$.

112 Extracted RNA for qRT-PCR were kept in $-80^{\circ} \mathrm{C}$ until further analysis by RT-LAMP. SARS$113 \mathrm{CoV} 2 \mathrm{RT}$-LAMP reactions were run at $65^{\circ} \mathrm{C}$ for $30 \mathrm{~min}$. Clinical sensitivity was calculated as 114 (number of true positives)/(number of true positives + number of false negatives) and clinical 
115 specificity was calculated as (number of true negatives) $/($ number of true negatives + number of

116 false positives) comparing to qRT-PCR. Ethical approval for this study was obtained from Medical

117 Research Ethics Committee (MREC) Ministry of Health Malaysia (NMRR-20-535-53855).

118

119

\section{Results}

The SARS-CoV2 RT-LAMP assay was able to detect one copy per reaction of SARS-CoV2 RNA in 30 mins while the LOD for qRT-PCR was five copies per reaction (Supplementary Table 2). The time taken for amplification did not change with or without the addition of HNB in the master mix (Supplementary Table 2). No cross-reactivity with other respiratory viruses (coronaviruses, adenovirus, human metapneumovirus, influenza A viruses, influenza B virus, parainfluenza virus (Supplementary Table 2).

127 The RT-LAMP assay demonstrated a 100\% sensitivity as all the 47 RNA samples that were positive by qRT-PCR were tested positive with RT-LAMP. None of the 42 qRT-PCR negative samples were positive for SARS-CoV2 using this assay (Supplementary Figure 3). No falsepositive reactions were noted (Supplementary Table 2, Supplementary Figure 4).

\section{Discussion}

133 The COVID-19 RT-LAMP reaction was sensitive enough to detect one copy of RNA per reaction,

134 5-fold better than real-time PCR. Addition of HNB to the LAMP reaction solution did not affect 135 the time taken for amplification. Several studies have found that LAMP out-performs qRT-PCR 136 for other viral infections $[6,10,11]$, which is consistent with our results. 
137 The analysis showed that the RT-LAMP developed is $100 \%$ specific and sensitive for the detection

138 of SARS-CoV2 with no false positives detected. The specificity and sensitivity levels of RT-

139 LAMP are comparable to real-time qRT-PCR methods as reported in other studies [12,13].

140 Encouragingly, the time required for confirmation of results by the RT-LAMP assay was less than

14130 mins, 2.5-fold faster than the time required by real-time PCR. Even including the RNA isolation

142 step, RT-LAMP assay can be completed in less than one hour, which is very short when compared

143 to that for real time qRT-PCR (two to two and a half hours).

144 Due to its high sensitivity, RT-LAMP is prone to aerosol contamination. LAMP assays can be

145 analysed by running an agarose gel or adding SYBR Green I. As it has an inhibitory effect, SYBR

146 Green I has to be added after completion of the LAMP reaction. Tubes used for RT-LAMP

147 reactions have to be opened for gel electrophoresis for addition of SYBR Green I, which can

148 contaminate the air and subsequent reactions. Therefore, in our study, to avoid contamination,

149 HNB dye was used to enable interpretation of the results by the naked eye, without requiring the 150 tubes to be opened. Addition of HNB to the LAMP reaction solution did not affect amplification

151 efficiency. This approach has been shown to be sensitive and simple for visual detection of turkey

152 coronavirus RNA in tissues and faeces [14].

153

\section{Conclusion}

155 There are several limitations in this study. First, the LOD of the SARS-CoV2 RT-LAMP assay

156 was not determined using serial dilutions of purified cell culture supernatant of SARS-CoV2 due

157 to the unavailability of BSL3 laboratory. Secondly, although we showed specificity when testing 158 against the most closely-related human coronavirus SARS-CoV, we were unable to obtain RNA 159 from other coronaviruses $\mathrm{HCoV}-229 \mathrm{E}, \mathrm{HCoV}-\mathrm{NL} 63$, and MERS-CoV for specificity analysis. 
160 These experiments should be included in the future to enhance the stringency of the RT-LAMP 161 assay.

162 In order to improve the efficiency of the RT-LAMP reaction, parameters such as heating 163 temperature, dNTP concentration, and reaction time can be further optimized. There is a high risk 164 of contamination due to the large amount of LAMP products, which often leads to false-positive 165 results. A typical molecular laboratory should be divided into at least three areas for sample 166 preparation, master mix preparation and product detection. However, this may not be available in 167 resource-limited countries. To avoid contamination, general cleaning practices such as 168 decontaminating all surfaces with 10-15\% sodium hypochlorite solution, followed by $70 \%$ 169 ethanol should be routinely carried out. It is highly recommended to adopt a closed end-point 170 detection method in order to avoid carry-over contamination. On top of that, additional mineral oil 171 inside the reaction tubes can reduce the risk of contamination. Sample processing time and cost 172 can be reduced by using direct pathogen detections without upstream RNA extraction by 173 commercial kits. Nie et al., (2012) [15] demonstrated that direct RT-LAMP assay can detect EV71 174 in heat-treated nasopharyngeal swab specimens.

175 Lastly, the sensitivity and specificity of the RT-LAMP assay can only be compared with qRT176 PCR as there is no true gold standard for SARS-CoV2 detection. It is possible that either test may 177 have misclassified the true result of some of the samples. It is also important to note that the LAMP 178 primers are highly specific, different primers may be needed for detection of different types of 179 mutations of the target gene in the future.

180 SARS-CoV-2 can also be detected in other biological samples such as sputum, bronchoalveolar 181 lavage fluid, feces and blood [16]. Future studies are expected to assess the feasibility of RT182 LAMP in detection of SARS-CoV2 RNA extracted from these biological samples. 
183 To conclude, an inexpensive, rapid, sensitive, and specific RT-LAMP assay was successfully 184 designed for SARS-CoV2 detection. The simplicity of RT-LAMP combined with rapid turnaround 185 time has shown it to be a valuable and applicable tool for the diagnosis of infectious diseases, 186 particularly in resource-limited countries. In addition, RT-LAMP can be easily adapted to point187 of-care diagnosis of COVID-19 as supported by a review written by Nguyen et al., (2020) [17] 188 and a non-peer-reviewed preprint by Zhang et. al., (2020) using seven clinical samples [18]. Early 189 and accurate diagnosis is crucial to identify patients with COVID-19 for prompt 190 institutionalization of infection control and public health measures, and when available, treatment. 191

\section{Acknowledgements}

193 We thank the Director General of the Ministry of Health Malaysia for his permission to publish 194 this paper. The following reagent was obtained through the NIH Biodefense and Emerging 195 Infections Research Resources Repository, NIAID, NIH: gamma-irradiated SARS-coronavirus, 196 NR-9547.

\section{References}

1. World Health Organization (2020a). WHO Health Emergency Dashboard, 200 https://extranet.who.int/publicemergency. Date accessed: 22 March 2020.

2. Centers for Disease Control (2020). Real-Time RT-PCR Panel for Detection 2019-Novel detection-instructions.pdf. Date accessed: 22 March 2020.

3. World Health Organization (2020b) Novel Coronavirus (2019-nCoV) technical guidance: Laboratory testing for 2019-nCoV in humans. 
206 https://www.who.int/emergencies/diseases/novel-coronavirus-2019/technical-

207 guidance/laboratory-guidance. Date accessed: 26 Mar 2020.

208 4. Corman VM, Landt O, Kaiser M, Molenkamp R, Meijer A, Chu DK, Bleicker T, Brünink S, 209 Schneider J, Schmidt ML, Mulders DG, Haagmans BL, van der Veer B, van den Brink S, 210 Wijsman L, Goderski G, Romette JL, Ellis J, Zambon M, Peiris M, Goossens H, Reusken C, 211 Koopmans MP, Drosten C. Detection of 2019 novel coronavirus (2019-nCoV) by real-time RT-PCR. Euro Surveill. 2020;25(3). 10.2807/1560-7917.ES.2020.25.3.2000045

5. Notomi T, Okayama H, Masubuchi H, Yonekawa T, Watanabe K, Amino N, Hase T. Loopmediated isothermal amplification of DNA. Nucleic Acids Res 2000;28 (12): E63.

6. Lau YL, Lai MY, Teoh BT, Abd-Jamil J, Johari J, Sam SS, Tan KK, AbuBakar S. Colorimetric Detection of Dengue by Single Tube Reverse-Transcription-Loop-Mediated Isothermal Amplification. PLoS ONE 2015;10(9):e0138694.

7. Wong YP, Othman S, Lau YL, Son R, Chee HY. Loop-mediated isothermal amplification (LAMP): a versatile technique for detection of micro-organisms. J App Microb 2017;124(3):626-643.

8. Lau YL, Lai MY, Fong MY, Jelip J, Mahmud R. Short Report: Loop-Mediated Isothermal 223 Amplification Assay for Identification of Five Human Plasmodium Species in Malaysia. Am J Trop Med Hyg 2016;94(2):336-9.

9. Zhang Y, Liu Q, Zhou B, Wang X, Chen S, Wang S. Ultra-sensitive chemiluminescence 225 226 imaging DNA hybridization method in the detection of mosquito-borne viruses and parasites. Parasites Vectors 2017;10: 44. 
227 228

229

230

231

232

233

234

235

236

237

238

239

240

241

242

243

244

245

246

247

248

10. Hu SF, Li M, Zhong LL, Lu SM, Liu ZX, Pu JY, Wen JS, Huang X. Development of reversetranscription loop-mediated isothermal amplification assay for rapid detection and differentiation of dengue virus serotypes 1-4. BMC Microbiol. 2015;15:265.

11. Zhao J, Feng R. Sensitive and rapid detection of Zika virus by loop-mediated isothermal amplification. Virus Genes 2019;55:43-50.

12. Reddy V, Ravi V, Desai A, Parida M, Powers AM, Johnson BW. Utility of IgM ELISA, TaqMan real-time PCR, reverse transcription PCR, and RT-LAMP assay for the diagnosis of Chikungunya fever. J Med Virol 2012;84(11):1771-1778.

13. Kwallah A, Inoue S, Muigai AW, Kubo T, Sang R, Morita K, Mwau M. A real-time reverse transcription loop-mediated isothermal amplification assay for the rapid detection of yellow fever virus. J Virol Methods 2013;193(1):23-27.

14. Cardoso TC, Ferrari HF, Bregano LC, Silva-Frade C, Rosa ACG, Andrade AL. Visual detection of turkey coronavirus RNA in tissues and feces by reverse-transcription loopmediated isothermal amplification (RT-LAMP) with hydroxynaphthol blue dye. Mol and Cell Prob. 2010;24(6):415-417.

15. Nie K, Qi S-x, Zhang Y, Luo L, Xie Y, Yang MJ, Zhang Y, Li J, Shen H, Li Q, Ma XJ. Evaluation of a Direct Reverse Transcription Loop-Mediated Isothermal Amplification Method without RNA Extraction for the Detection of Human Enterovirus 71 Subgenotype C4 in Nasopharyngeal Swab Specimens. PLoS ONE 2012;7(12): e52486.

16. Wang W, Xu Y, Gao R, Lu R, Han K, Wu G, Tan W. Detection of SARS-CoV-2 in Different Types of Clinical Specimens. JAMA. Published online March 11, 2020. doi:10.1001/jama.2020.3786 
249 17. Nguyen T, Bang DD, Wolff A. 2019 Novel Coronavirus Disease (COVID-19): Paving the 250 Road for Rapid Detection and Point-of-Care Diagnostics. Micromachines 2020:11(3):306.

251 18. Zhang Y, Nelson O, Jin X, Luo S, Raphael N, Wei H, Nathan T. Rapid Molecular Detection 252 of SARS-CoV-2 (COVID-19) Virus RNA Using Colorimetric LAMP [Preprint]. Available at: 253 https://www.medrxiv.org/content/10.1101/2020.02.26.20028373v1.full (Accessed: 4 April $2542020)$. 


\section{Figure 1}

Asessment of RT-LAMP results based on hydroxynaphthol blue visualization of color change.

A positive reaction indicated by sky blue colour is seen in tubes 1-2 and the positive control (PC), while a violet colour indicates a negative reaction in tubes 4-5 and the non-template negative control (NC).

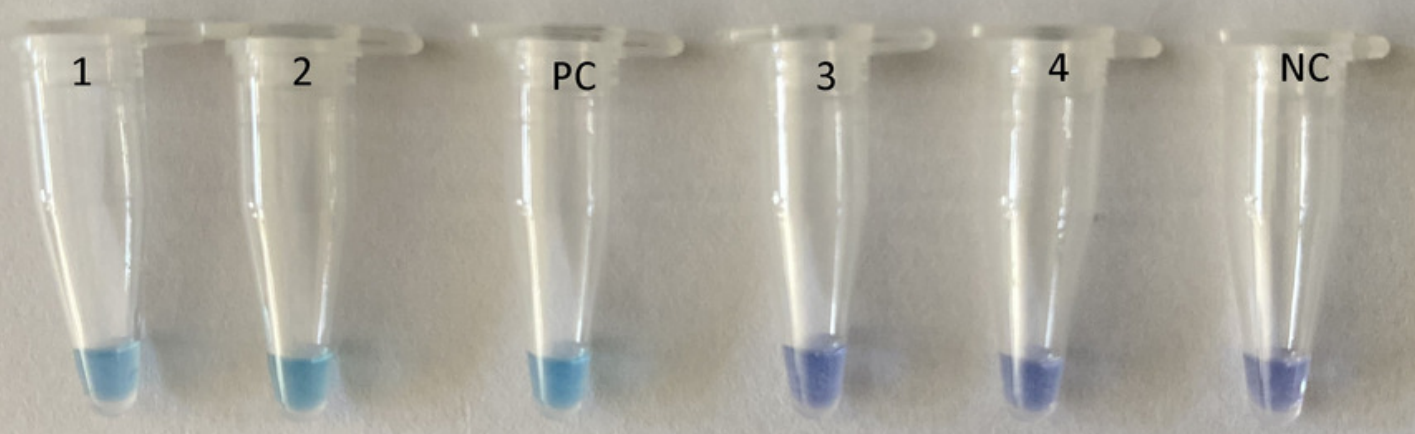


Figure 2

qRT-PCR results for SARS-CoV2 detection.

The chart was generated by plotting relative fluorescence (RFU) vs. cycle number, with each colored line representing one sample. Seven positive samples and a positive control (PC) are shown with cycle threshold levels between 18 to 40 cycles.

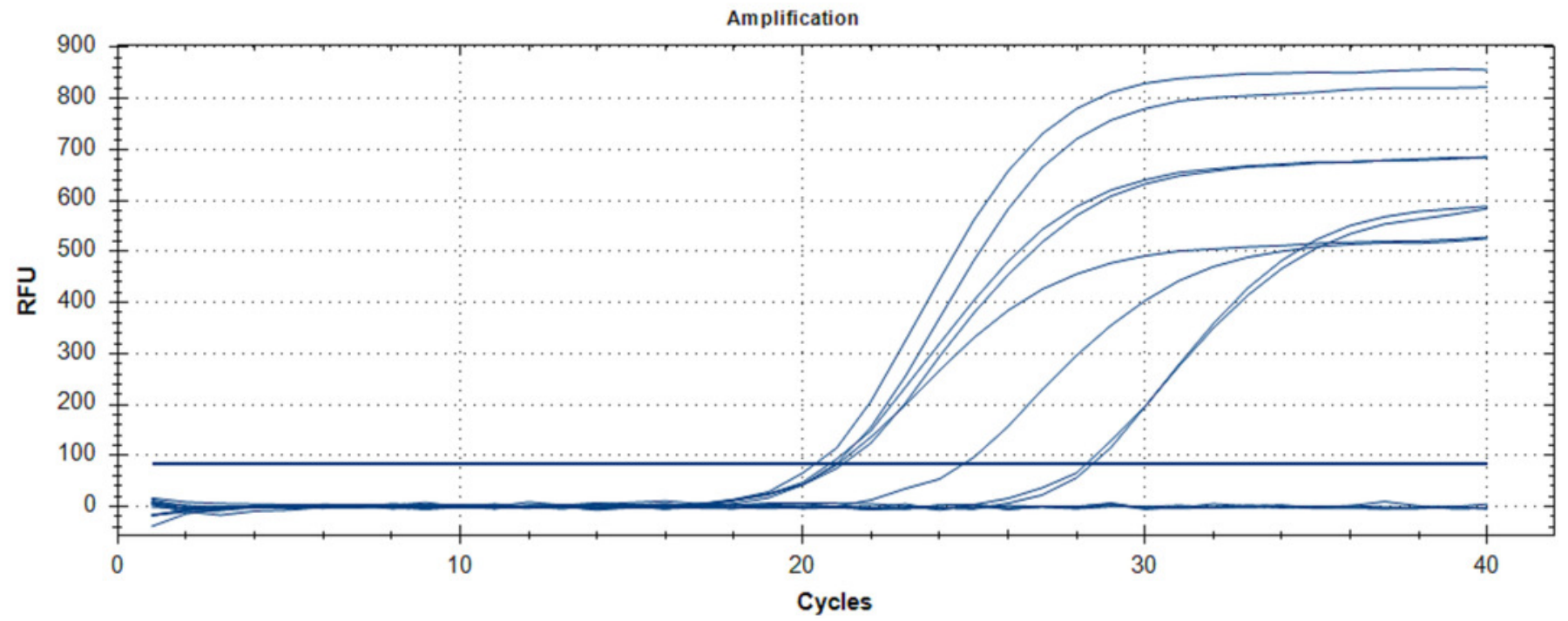

